\title{
Towards Digital Cohomology ${ }^{\star}$
}

\author{
Rocio Gonzalez-Diaz and Pedro Real \\ Applied Math Dept., University of Seville, Spain, \\ \{rogodi, real\}@us.es, \\ http://www.us.es/gtocoma
}

\begin{abstract}
We propose a method for computing the $\mathbf{Z}_{2}-$ cohomology ring of a simplicial complex uniquely associated with a three-dimensional digital binary-valued picture $I$. Binary digital pictures are represented on the standard grid $\mathbf{Z}^{3}$, in which all grid points have integer coordinates. Considering a particular 14-neighbourhood system on this grid, we construct a unique simplicial complex $K(I)$ topologically representing (up to isomorphisms of pictures) the picture $I$. We then compute the cohomology ring on $I$ via the simplicial complex $K(I)$. The usefulness of a simplicial description of the digital $\mathbf{Z}_{2}-$ cohomology ring of binary digital pictures is tested by means of a small program visualizing the different steps of our method. Some examples concerning topological thinning, the visualization of representative generators of cohomology classes and the computation of the cup product on the cohomology of simple 3D digital pictures are showed.
\end{abstract}

Keywords: Digital topology, chain complexes, cohomology ring.

\section{Introduction}

The homology groups (given in terms of number of connected components, holes and cavities in the digital picture), the digital Euler characteristic or the digital fundamental group are well-known operations in Digital Topology [15,10]. All of them can be considered as translations into the discrete setting of classical continuous topological invariants. In order to prove that a digital topology operation $\pi_{D}$ (associated with a continuous operation $\pi_{C}$ ) correctly reflects the topology of digital pictures considered as Euclidean spaces, the main idea is to associate a "continuous analog" $C(I)$ with the digital picture $I$. In most cases, each binary digital picture $I$ is associated with a polyhedron $C(I)$ 101191]). It is clear that $C(I)$ "fills the gaps" between black points of $I$ in a way that strongly depends on the grid and adjacency relations chosen for the digital picture $I$. Recent attempts to enrich the list of computable digital topological invariants in such a way can be found in 8 .

In this paper, we will consider binary digital pictures $I=\left(\mathbf{Z}^{3}, 14,14, B\right)$, having the standard lattice $\mathbf{Z}^{3}$ as the underlying grid and fixing a special $14-$ adjacency for both the points of $B$ and the points of its complement. Our binary digital picture space (or, briefly, DPS) is regular and isomorphic to the

\footnotetext{
* Partially supported by the PAICYT research project FQM-296 from Junta de Andalucía (Spain).
} 
well-known DPS called 3-d body-centered cubic grid [11. Starting from a picture $I$, we construct, in a straightforward way, a simplicial complex $K(I)$ based on the triangulation of the Euclidean 3-space determined by the previous 14neighbourhood relation: The $i$-simplices of $K(I)(i \in\{0,1,2,3\})$ are constituted by the different sets of $i$ 14-neighbour black points in $I$ (analogously, we can construct another simplicial complex whose $i$-simplices are the different sets of $i$ 14-neighbour white points in $I$ ). We do not take care of the orientation of the simplices due to the fact that we are interested in computing the mod 2 cohomology. Since an isomorphism of pictures is equivalent to a simplicial homeomorphism of the corresponding simplicial representations, we are able to define the digital cohomology ring $H^{*}\left(I ; \mathbf{Z}_{2}\right)$ as the cohomology ring $H^{*}\left(K(I) ; \mathbf{Z}_{2}\right)$. In this simplicial setting and using the technique of simplicial collapses [5], we topologically thin $K(I)$, obtaining a smaller simplicial complex $M_{\text {top }} K(I)$. The following step is the computation of the cohomology ring $H^{*}\left(M_{\text {top }} K(I) ; \mathbf{Z}_{2}\right)$. Since $H^{*}\left(M_{\text {top }} K(I) ; \mathbf{Z}_{2}\right)$ is isomorphic to $H^{*}\left(K(I) ; \mathbf{Z}_{2}\right)$, the information obtained in this way can be used for "topologically" classifying (up to isomorphisms of pictures) and distinguishing (up to cohomology ring level) 3-d binary digital pictures. A small program, called EditCup, for editing binary digital pictures and visualizing cohomology aspects of them has been designed by the authors and developed by others 1. This software allows us to test in some simple examples the potentiality and topological acuity of our method.

\section{Simplicial Representation of 3D Pictures}

We follow the terminology given in 11] for representing binary digital pictures. A $3 D$ binary digital picture space (or, briefly, DPS) is a triple $(V, \beta, \omega)$, where $V$ is the set of grid points in a $3-\mathrm{d}$ grid and each of $\beta$ and $\omega$ is a set of closed straight line segments joining pairs of points in $V$. The set $\beta$ (resp. the set $\omega)$ determines the neighbourhood relations between black points (resp. white points) in the grid. An isomorphism of a DPS $\left(V_{1}, \beta_{1}, \omega_{1}\right)$ to a $\operatorname{DPS}\left(V_{2}, \beta_{2}, \omega_{2}\right)$ is a homeomorphism $h$ of the Euclidean 3 -space to itself such that $h$ maps $V_{1}$ onto $V_{2}$, each $\beta_{1}$-adjacency onto a $\beta_{2}$-adjacency and each $\omega_{1}$-adjacency onto an $\omega_{2}$-adjacency, and $h^{-1}$ maps each $\beta_{2}$-adjacency onto a $\beta_{1}$-adjacency and each $\omega_{2}$ adjacency onto an $\omega_{1}$-adjacency. A $3 D$ digital binary picture is a quadruple $I=(V, \beta, \omega, B)$, where $(V, \beta, \omega)$ is a DPS and $B$ (the set of black points) is a finite subset of $V$. An isomorphism of a picture $I_{1}=\left(V_{1}, \beta_{1}, \omega_{1}, B_{1}\right)$ to a picture $I_{2}=\left(V_{2}, \beta_{2}, \omega_{2}, B_{2}\right)$ is an isomorphism of the DPS $\left(V_{1}, \beta_{1}, \omega_{1}\right)$ to the DPS $\left(V_{2}, \beta_{2}, \omega_{2}\right)$ that maps $B_{1}$ onto $B_{2}$.

The DPS used in this paper, that we call $(14,14)-\mathrm{DPS}$, is $\left(\mathbf{Z}^{3}, 14,14\right)$, in which the underlying grid is the set of points with integer coordinates in the Euclidean 3-space $E^{3}$ and the 14-neighbours of a grid point (black or white) with integer coordinates $(x, y, z)$ are: $(x \pm 1, y, z),(x, y \pm 1, z),(x, y, z \pm 1)$,

1 The 1 st version was programmed by J.M. Berrio, F. Leal and M.M. Maraver. The 2nd version was programmed by F.Leal. This program has been already presented in [2]. http://www.us.es/gtocoma/editcup.zip. 
$(x+1, y-1, z),(x-1, y+1, z),(x+1, y, z-1),(x-1, y, z+1),(x, y+1, z-1)$, $(x, y-1, z+1),(x+1, y+1, z-1),(x-1, y-1, z+1)$. Nevertheless, the $14-$ adjacency for digital pictures has been usually defined in a $3-\mathrm{d}$ body-centered cubic grid (BCC grid) [11]: The grid points are the points $(a, b, c) \in \mathbf{Z}^{3}$ such that $a \equiv b \equiv c(\bmod 2)$. The 14-neighbours of a grid point $p$ with coordinates $(a, b, c)$ are: $(a \pm 2, b, c),(a, b \pm 2, c),(a, b, c \pm 2),(a \pm 1, b \pm 1, c \pm 1)$. The (14,14)-DPS and the BCC grid are isomorphic DPSs: a grid point $(x, y, z)$ of the $(14,14)-\mathrm{DPS}$ can be associated to the point $(x+y+2 z,-x+y,-x-y)$ of the BBC grid.
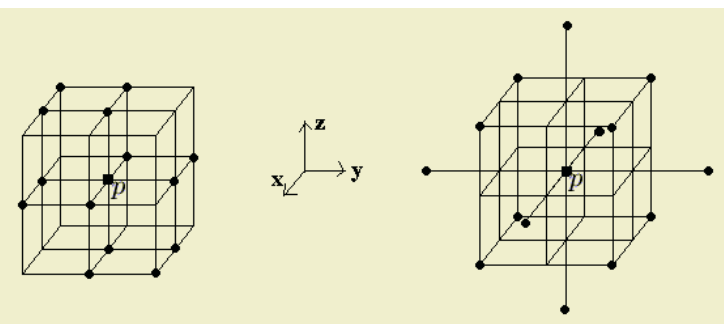

Fig. 1. The 14-neighbours of a grid point $p$ of the $(14,14)-$ DPS (on the left) and the BCC grid (on the right).

\section{An Approach to Digital Cohomology Ring}

Given a binary digital picture $I=\left(\mathbf{Z}^{3}, 14,14, B\right)$ on the $(14,14)$-DPS, we can uniquely associate with it a 3 -dimensional simplicial complex $K(I)$ that we call the simplicial representation of the digital picture $I$. The vertices (or $0-$ simplices) of $K(I)$ are the points of $I$. The edges, triangles and tetrahedra are formed joining two, three and four 14-neighbour points of $B$, respectively. This naive simplicial construction, together with the satisfactory algorithmic solution presented here to the problem of the computation of cohomology operations on finite simplicial complexes, will allow to "cohomologically control" the digital picture $I$ (up to isomorphisms of pictures). Before explaining in detail the different steps of our method, we will enunciate the following theorem whose proof is straightforward and left to the reader.

Theorem 1. Two binary digital pictures, $I_{1}=\left(\mathbf{Z}^{3}, 14,14, B_{1}\right)$ and $I_{2}=\left(\mathbf{Z}^{3}, 14\right.$, $\left.14, B_{2}\right)$, are isomorphic if and only if the simplicial representations $K\left(I_{1}\right)$ and $K\left(I_{2}\right)$ are simplicially homeomorphic.

This last result allows us to define the following notion:

Definition 1. Given a binary digital picture $I=\left(\mathbf{Z}^{3}, 14,14, B\right)$, the digital $\mathbf{Z}_{2}$ cohomology ring of $I$ is defined as the $\mathbf{Z}_{2}$-cohomology ring of $K(I)$.

Since the simplicial complexes considered in this paper are embedding in $\mathbf{R}^{3}$ then homology groups are torsion free (moreover, the possible non-null homology groups are $H_{0}(K), H_{1}(K)$ and $H_{2}(K)$ ). Therefore, homology and cohomology are isomorphic. The $q$-Betti number is the rank of the $q$ th homology group. 
In general, the 0th Betti number is the number of connected components, the 1st and 2nd Betti numbers have intuitive interpretations as the number of independent non-bounding loops and the number of independent non-bounding shells. Since the Betti numbers are independent of the group of coefficients we consider, throughout the paper, the ground ring is $\mathbf{Z}_{2}$.

In the next three subsections, we will reinterpret classical methods in Algebraic Topology and Homological Algebra in terms of chain contractions [12] that will enable us to design an algorithm for computing the cohomology rings of binary digital pictures. Now, the previous reading of the appendix is strongly recommended if the reader is not familiar with the concepts from Algebraic Topology presented in this section. Let us emphasize that a fundamental notion here is that of chain contraction:

Definition 2. A chain contraction from a chain complex $\mathcal{C}$ to another chain complex $\mathcal{C}^{\prime}$ is a set of three homomorphisms $(f, g, \phi)$ such that:

$-f: \mathcal{C} \rightarrow \mathcal{C}^{\prime}$ and $g: \mathcal{C}^{\prime} \rightarrow \mathcal{C}$ are chain maps.

- $f g$ is the identity map of $\mathcal{C}^{\prime}$.

$-\phi: \mathcal{C} \rightarrow \mathcal{C}$ is a chain homotopy of the identity map $i d_{\mathcal{C}}$ of $\mathcal{C}$ to gf, that is, $\phi \partial+\partial \phi=i d_{\mathcal{C}}+g f$.

Important properties of chain contractions are that $\mathcal{C}^{\prime}$ has fewer or the same number of generators than $\mathcal{C}$, and $\mathcal{C}$ and $\mathcal{C}^{\prime}$ have isomorphic homology groups. We will also use the following notation: let $a$ be a chain and $b$ an element of $a$. We denote by $(a ; b)$ the new chain obtained replacing $b$ by a variable $x$ and solving the equation $a=0$ for the variable $x$.

\subsection{Topological Thinning}

Topological thinning is an important preprocessing operation in Image Processing. The aim is to shrink a digital picture to a smaller, simpler picture which retains a lot of the significant information of the original.Then, further processing or analysis can be performed on the shrunken picture.

In our approach, a 3D binary digital picture is directly converted into a $3 \mathrm{D}$ simplicial complex. There is a well-known process for thinning a simplicial complex using simplicial collapses [3]. Suppose $K$ is a simplicial complex, $\sigma \in K$ is a maximal simplex and $\sigma^{\prime}$ is a free facet of $\sigma$. Then, $K$ simplicially collapses onto $K-\left\{\sigma, \sigma^{\prime}\right\}$. An important property of this process is that there exists an explicit chain contraction from $C(K)$ to $C\left(K-\left\{\sigma, \sigma^{\prime}\right\}\right)$ [5]. More generally, a simplicial collapse is any sequence of such operations. A thinned simplicial complex $M_{\text {top }} K$ is a subcomplex of $K$ with the condition that all the faces of the maximal simplices of $M_{\mathrm{top}} K$ are shared. Then, it is obvious that it is no longer possible to collapse. There is also an explicit chain contraction from $C(K)$ to $C\left(M_{\text {top }} K\right)$. In particular, recall that this means that the (co)homology of $K$ and $M_{\text {top }} K$ are isomorphic.

The following algorithm computes $M_{\text {top }} K$ and a chain contraction ( $f_{\text {top }}, g_{\text {top }}$, $\left.\phi_{\text {top }}\right)$ from $C(K)$ to $C\left(M_{\text {top }} K\right)$. Initially, $M_{\text {top }} K=K$. 
While there exists a maximal simplex $\sigma$ with a free facet $\sigma^{\prime}$ do $M_{\text {top }} K:=M_{\text {top }} K-\left\{\sigma, \sigma^{\prime}\right\}, f_{\text {top }}(\sigma):=0, ; \phi_{\text {top }}(\sigma):=0$, $f_{\text {top }}\left(\sigma^{\prime}\right):=f_{\text {top }}\left(\partial \sigma ; \sigma^{\prime}\right), \phi_{\text {top }}\left(\sigma^{\prime}\right):=\sigma+\phi_{\text {top }}\left(\partial \sigma ; \sigma^{\prime}\right)$; if $\sigma \in M_{\text {top }} K$, then $f_{\text {top }}(\sigma):=\sigma, g_{\text {top }}(\sigma):=\sigma$ and $\phi_{\text {top }}(\sigma):=0$. End.

\section{2 "Algebraic Thinning"}

Having obtained the simpler thinned complex $M_{\mathrm{top}} K(I)$, we next compute its homology. The computation of a chain contraction $\left(f_{\mathrm{alg}}, g_{\mathrm{alg}}, \phi_{\mathrm{alg}}\right)$ from the chain complex $C\left(M_{\text {top }} K(I)\right)$ to its homology can be considered as a thinning, at algebraic level, of $C\left(M_{\text {top }} K(I)\right)$ (for this reason we call it "algebraic thinning"). We compute $\left(f_{\text {alg }}, g_{\text {alg }}, \phi_{\text {alg }}\right)$ interpreting the "incremental algorithm" 44 for computing homology groups in $\mathbf{R}^{3}$ in terms of chain homotopies. This procedure is essential for us in order to calculate the cohomology ring of $I$.

Let $\left(\sigma_{1}, \ldots, \sigma_{m}\right)$ be a sorted set of all the simplices of a given simplicial complex $L$ with the property that any subset $\left\{\sigma_{1}, \ldots, \sigma_{i}\right\}, i \leq m$, is a subcomplex of $L$. The algorithm computes a chain complex $\mathcal{C}$ with set of generators $h$, and a chain contraction $\left(f_{\text {alg }}, g_{\text {alg }}, \phi_{\text {alg }}\right)$ from $C(L)$ to $\mathcal{C}$. Initially, $h$ is empty. In the step $i$ th of the algorithm, the simplex $\sigma_{i}$ is added to the subcomplex $\left\{\sigma_{1}, \ldots, \sigma_{i-1}\right\}$ and then, a homology class is created or destroyed. If $f_{\text {alg }} \partial\left(\sigma_{i}\right)=0$ then $\sigma_{i}$ "creates" the class $\alpha_{i}$. Otherwise, $\sigma_{i}$ "destroys" one homology class involved in the expression of $f_{\text {alg }} \partial\left(\sigma_{i}\right)$. At the end of the algorithm, $\mathcal{C}$ is a chain complex isomorphic to the homology of $L$. The pseudocode of the algorithm is:

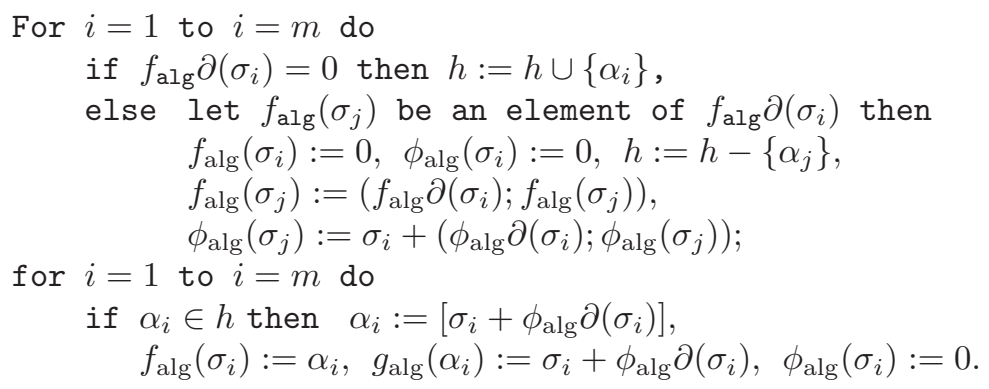

End.

Recall that the output of the algorithm is a chain contraction $\left(f_{\text {alg }}, g_{\text {alg }}, \phi_{\text {alg }}\right)$ from $C(L)$ to $H(L)$, allowing us to determine both a representative cycle for each homology class and the homology class for each cycle. Moreover, for any $q$-boundary $a$ on $L$ we can obtain a $(q+1)$-chain $a^{\prime}=\phi_{\text {alg }}(a)$ on $L$ such that $a=\partial\left(a^{\prime}\right)$. The algorithm runs in time at most $O\left(m^{3}\right)$ if $L$ has $m$ simplices.

The idea of computing a contraction from a chain complex to its homology has also been used in [67. In [6] the computation of the contraction is based on the transcription of the reduction algorithm [14, p. 58] and it is used for computing primary and secondary cohomology operations. 
We can compose the chain contraction $\left(f_{\text {top }}, g_{\text {top }}, \phi_{\text {top }}\right)$ from $C(K(I))$ to $C\left(M_{\text {top }} K(I)\right)$, described in the section above, with that $\left(f_{\text {alg }}, g_{\text {alg }}, \phi_{\text {alg }}\right)$ from $C\left(M_{\text {top }} K(I)\right)$ to $H\left(M_{\text {top }} K(I)\right)$ (which is isomorphic to $\left.H(K(I))\right)$. We then obtain a new chain contraction [12] $\left(f_{\text {alg }} f_{\text {top }}, g_{\text {top }} g_{\text {alg }}, \phi_{\text {top }}+g_{\text {top }} \phi_{\text {alg }} f_{\text {top }}\right)$ from $C(K(I))$ to $H(K(I))$.

Example 1. Let $I$ be the digital picture showed in Figure 3. The non-null images of the component morphisms of a chain contraction $\left(f_{\text {alg }}, g_{\text {alg }}, \phi_{\text {alg }}\right)$ from $C(K(I))$ to $H(K(I))$ obtained using the algorithm explained above are:

\begin{tabular}{c|c|c|c|c}
$K$ & $h$ & $f_{\text {alg }}$ & $g_{\text {alg }}$ & $\phi_{\text {alg }}$ \\
\hline$\langle 1\rangle$ & $\alpha_{1}$ & $\alpha_{1}$ & $\langle 1\rangle$ & 0 \\
$\langle 2\rangle$ & & $\alpha_{1}$ & & $\langle 1,7\rangle+\langle 6,7\rangle+\langle 5,6\rangle+\langle 4,5\rangle+\langle 3,4\rangle+\langle 2,3\rangle$ \\
$\langle 3\rangle$ & & $\alpha_{1}$ & & $\langle 1,7\rangle+\langle 6,7\rangle+\langle 5,6\rangle+\langle 4,5\rangle+\langle 3,4$ \\
$\langle 4\rangle$ & & $\alpha_{1}$ & & $\langle 1,7\rangle+\langle 6,7\rangle+\langle 5,6\rangle+\langle 4,5\rangle$ \\
$\langle 2,4\rangle$ & & 0 & & $\langle 2,3,4\rangle$ \\
$\langle 5\rangle$ & & $\alpha_{1}$ & & $\langle 1,7\rangle+\langle 6,7\rangle+\langle 5,6\rangle$ \\
$\langle 6\rangle$ & & $\alpha_{1}$ & & $\langle 1,7\rangle+\langle 6,7\rangle$ \\
$\langle 7\rangle$ & & $\alpha_{1}$ & & 0
\end{tabular}

Where $a=\langle 1,2\rangle+\phi(\langle 2\rangle)$. Therefore, $H_{0}(I) \simeq \mathbf{Z}_{2}, H_{1}(I) \simeq \mathbf{Z}_{2}$ and $H_{2}(I)=0$.

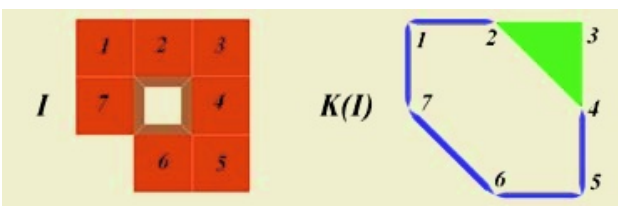

Fig. 2. A digital picture $I$ and its simplicial representation $K(I)$.

\subsection{Computing the Digital $\mathrm{Z}_{2}$-Cohomology Ring}

After applying topological and algebraic thinning to the simplicial representation $K$ of a binary digital picture $I$ in order, we are able to compute the multiplication table on the cohomology.

Let $(f, g, \phi)$ be a contraction from $C(K)$ to $H(K)$. Observe that if $\gamma \in H_{q}(K)$ then $\gamma^{*}: H_{q}(K) \rightarrow \mathbf{Z}_{2}$, defined by $\gamma^{*}(\omega)=1$ if $\omega=\gamma$ and $\gamma^{*}(\omega)=0$ otherwise, is a cohomology class of $K$. Moreover, $\gamma^{*} f: C_{q}(K) \rightarrow \mathbf{Z}_{2}$ is a representative cocycle of $\gamma^{*}$. Let $\left\{\alpha_{1}, \ldots, \alpha_{p}\right\}$ and $\left\{\beta_{1}, \ldots, \beta_{q}\right\}$ be sets of generators of $H_{1}(K)$ and $H_{2}(K)$ then, $\left\{\alpha_{1}^{*}, \ldots, \alpha_{p}^{*}\right\}$ and $\left\{\beta_{1}^{*}, \ldots, \beta_{q}^{*}\right\}$ are sets of generators of $H^{1}(K)$ and $H^{2}(K)$. The cohomology ring of $K$ is computed as follows:

$$
\begin{aligned}
& \text { For } i=1 \text { to } i=p \text { do } \\
& \quad \text { for } j=i \text { to } j=p \text { do } \sum_{k=1}^{q}\left(\left(\alpha_{i}^{*} f \smile \alpha_{j}^{*} f\right)\left(g \beta_{k}\right)\right) \cdot \beta_{k}^{*} \text {. }
\end{aligned}
$$
End. 


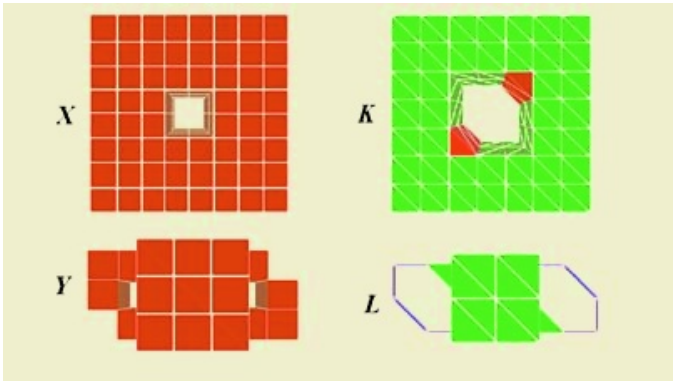

Fig. 3. The pictures $X$ and $Y$ and their simplicial representations $K$ and $L$.

Given a binary digital picture $I$, the total algorithm for computing the cohomology ring of $I$ runs in time at most $O\left(m^{6}\right)$ if $K(I)$ has $m$ simplices.

As we have said in Section 1, in order to show an example of the computation and visualization of the cohomology ring of simple 3D binary digital pictures, we expose a small prototype called EditCup. We use a free program for building 3D words. In our case, a world is a particular 3D simplicial complex $K$ representing a digital picture $I$ considering the 14-adjacency. A way for distinguishing the different maximal simplices of the simplicial complex associated with a simplicial representation is by using different colours: red for tetrahedra, green for triangles, blue for edges, and black for vertices. For visualizing (co)chains, the simplices on which a given (co)chain is non-null, are lighted in a different color. On the other hand, the "visualization" of any $\mathbf{Z}_{2}-(\mathrm{co})$ homology class on the original binary digital picture $I$ is given by lighting the points of $I$ such that the corresponding vertices span simplices on which the representative cochain of this class (obtained using our algorithm) is non-null.

Let us consider now the following pictures (see Figure 4): the torus (the picture $X$ ) and the wedge of two topological circles and a topological 2-sphere (the picture $Y$ ). In order to compute the cup product, we need the simplicial representation $K$ and $L$ of $X$ and $Y$, respectively (see Figure 4). It is clear that the (co)homology groups of $X$ are isomorphic to those of $Y$. They are $\mathbf{Z}_{2}, \mathbf{Z}_{2} \oplus \mathbf{Z}_{2}$ and $\mathbf{Z}_{2}$ in dimension 0,1 and 2 , respectively. Let us denote by $a_{1}, a_{2}$ and $a_{3}$ the representative cycles of the classes in $H_{1}(K)$ and $H_{2}(K)$, respectively; and by $a_{1}^{\prime}$, $a_{2}^{\prime}$ and $a_{3}^{\prime}$ the same in $H_{1}(L)$ and $H_{2}(L)$. We show the visualization of this cycles in Figure 5. In Figure 6 we show the two representative cocycles $u$, $v$ generating $H^{1}(K)$ and the cup product $w=u \smile v$ which is a representative cocycle of $H^{2}(K)$. If we consider now the representative cocycles $u^{\prime}$ and $v^{\prime}$ generating $H^{1}(L)$, and $w^{\prime}$ generating $H^{2}(L)$ then $\left[u^{\prime}\right] \smile\left[u^{\prime}\right]=\left[v^{\prime}\right] \smile\left[v^{\prime}\right]=\left[u^{\prime}\right] \smile\left[v^{\prime}\right]=0$. We conclude that $X$ and $Y$ are not isomorphic.

Let us note that this multiplication table for the cohomology ring of $K$ is not suitable in general for topological classification tasks, due to the fact that determining whether two rings are isomorphic or not by means of its respective multiplication tables is an extremely difficult computational question. In order to avoid this problem, we can put the information of the cup product in a matrix form $M$ (pairs of cohomology classes of dimension $1 \times$ cohomology classes of 


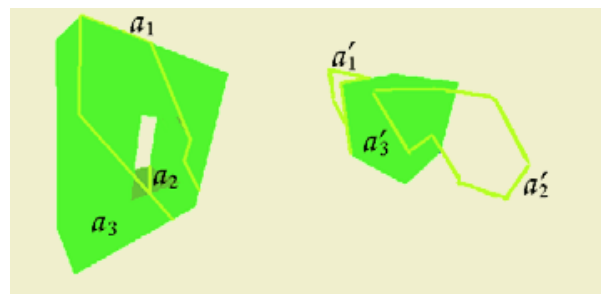

Fig. 4. The cycles $a_{1}, a_{2}$ and $a_{1}^{\prime}, a_{2}^{\prime}$ (in yellow); and $a_{3}$ and $a_{3}^{\prime}$ (in green).

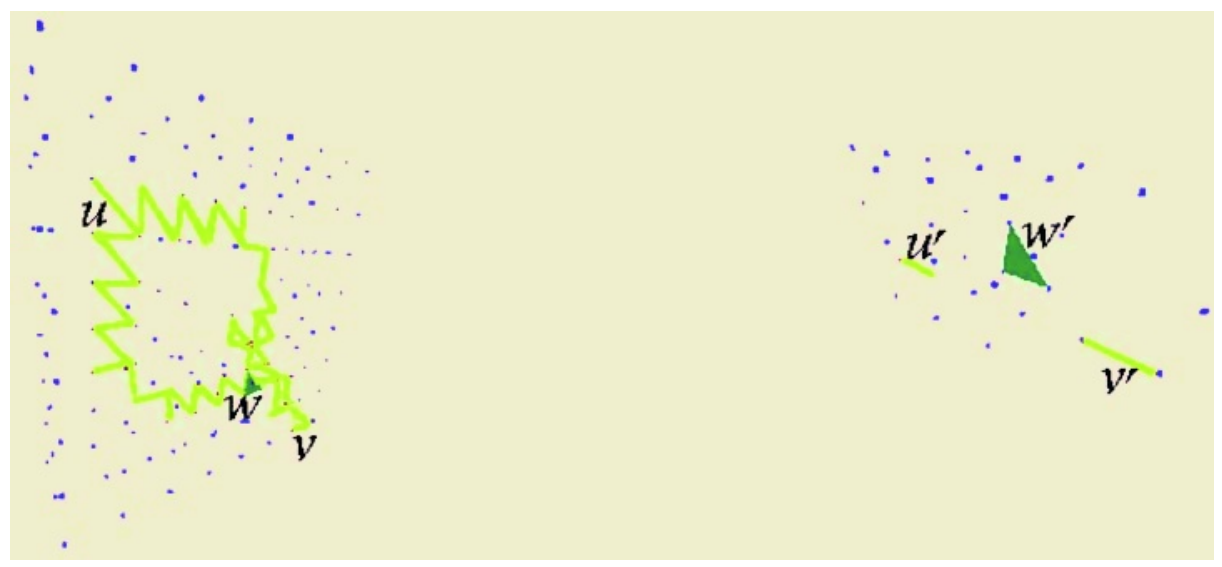

Fig. 5. The cocycles $u, v$ and $u^{\prime}, v^{\prime}$ (in yellow); and $w$ and $w^{\prime}$ (in green).

dimension 2). From the diagonalization $D$ of the matrix $M$, a first cohomology invariant $H B_{1}(I)$ appropriate for distinguishing non-isomorphic binary digital pictures with isomorphic (co)homology groups appears.

Definition 3. Given a 3D binary digital picture $I$, the cohomology invariant $H B_{1}(I)$ is defined as the rank of the matrix $M$.

For example, the matrices corresponding to the cohomology rings of the pictures $X$ and $Y$ are:

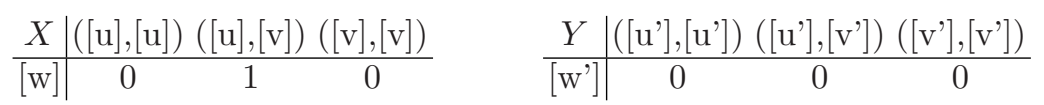

Therefore, $H B_{1}(X)=1$ and $H B_{1}(Y)=0$.

In fact, more complicated topological invariants can be derived from the cohomology ring in a similar way. Constructing these invariants is a very technical matter and we will study it in detail in the near future. Nevertheless, we will confine ourselves to say that these topological numbers can be directly generated from the homology of a well-known chain complex in Homological Algebra: the reduced bar construction of an algebra [12. 


\section{References}

1. Ayala R., Domínguez E., Francés A.R., Quintero A.: Homotopy in Digital Spaces. DGCI 2000 LNCS, Springer-Verlag 1953 (2000) 3-14

2. Berrio J.M., González-Díaz R., Leal F., López M.M., Real P.: Visualizing Cohomology Aspects of 3D Objects. Proc. of the 6th Asian Tech. Conf. in Math. (2001) 459-468.

3. Björner A.: Topological Methods. Handbook on Combinatorics. Elsevier Sci., 2 (1995) 1819-1872

4. Delfinado C.J.A., Edelsbrunner H.: An Incremental Algorithm for Betti Numbers of Simplicial Complexes on the 3-Sphere. Comput. Aided Geom. Design 12 (1995) $771-784$

5. Forman R.: Combinatorial Differential Topology and Geometry. New Perspective in Geom. Combinatorics. MSRI Public. 8 (1999) 177-206

6. González-Díaz R., Real P.: Computation of Cohomology Operations on Finite Simplicial Complexes. Homology, Homotopy and Applications 5 (2) (2003) 83-93

7. González-Díaz R., Real P.: Geometric Objects and Cohomology Operations. Proc. of the 5th Workshop on Computer Algebra in Scientific Computing (2002) 121-130

8. Kenmochi Y., Imiya A.: Discrete Polyhedrization of Lattice Point Set. Digital and Image Geometry, LNCS, Springer-Verlag 2243 (2001) 150-162

9. Khalimsky E.D., Kopperman R.D., Meyer P.R.: Computer Graphics and Connected Topologies on Finite Ordered Sets. Topology and Appl. 36 (1990) 1-17

10. Kong T.Y.: A digital Fundamental Group. Comput. Graphics 13 (1989) 159-166

11. Kong T.Y., Roscoe A.W., Rosenfeld A.: Concepts of Digital Topology. Topology and its Applications 8 (1992) 219-262

12. MacLane S.: Homology. Classic in Math., Springer-Verlag (1995)

13. Kovalevsky V.A.: Discrete Topology and Contour Definition. Pattern Recognition Letter 2 (1984) 281-288

14. Munkres J.R.: Elements of Algebraic Topology. Addison-Wesley Co. (1984)

15. Rosenfeld A.: 3D Digital Topology. Inform. and Control 50 (1981) 119-127

\section{Appendix: Basic Notions From Algebraic Topology}

In this section we briefly explain the main concepts from Algebraic Topology we use in this paper. Our terminology follows Munkres book [14].

The four types of non-empty simplices in $\mathbf{R}^{3}$ are: a 0 -simplex which is a vertex, a 1-simplex which is an edge, a 2-simplex which is a triangle and a 3 simplex which is a tetrahedron. Considering an ordering on a vertex set $V$, a $q$-simplex with vertices $v_{0}<\cdots<v_{q}$ in $V$ is denoted by $\left\langle v_{0}, \ldots, v_{q}\right\rangle$. If $i<q$, an $i$-face of $\sigma$ is an $i$-simplex whose vertices are in the set $\left\{v_{0}, \ldots, v_{q}\right\}$. A facet of $\sigma$ is a $(q-1)$-face of it. A simplex is shared if it is a face of more than one simplex. Otherwise, the simplex is free if it belongs to one higher dimensional simplex, and maximal if it does not belong to any.

A simplicial complex $K$ is a collection of simplices such that every face of a simplex of $K$ is in $K$ and the intersection of any two simplices of $K$ is a face of each of them or empty. The set of all the $q$-simplices of $K$ is denoted by $K^{(q)}$. A subset $K^{\prime} \subseteq K$ is a subcomplex of $K$ if it is a simplicial complex itself. Let $K$ and $L$ be simplicial complexes and let $|K|$ and $|L|$ be the subsets of $\mathbf{R}^{3}$ that 
are the union of simplices of $K$ and $L$, respectively. Let $f: K^{(0)} \rightarrow L^{(0)}$ be a map such that whenever the vertices $v_{0}, \ldots, v_{n}$ of $K$ span a simplex of $K$, the points $f\left(v_{0}\right), \ldots, f\left(v_{n}\right)$ are vertices of a simplex of $L$. Then $f$ can be extended to a continuous map $g:|K| \rightarrow|L|$ such that if $x=\sum t_{i} v_{i}$ then $g(x)=\sum t_{i} f\left(v_{i}\right)$. The map $g$ is called a simplicial homeomorphism if $f$ is bijective and the points $f\left(v_{0}\right), \ldots, f\left(v_{n}\right)$ always span a simplex of $L$.

A chain complex $\mathcal{C}$ is a sequence $\cdots C_{q+1} \stackrel{\partial_{q+1}}{\longrightarrow} C_{q} \stackrel{\partial_{q}}{\longrightarrow} C_{q-1} \cdots$ of abelian groups $C_{i}$ and homomorphisms $\partial_{i}$, indexed with the integers, such that for all $q$, $\partial_{q} \partial_{q+1}=0$. A $q$-chain $a \in C_{q}$ is called a $q-$ cycle if $\partial_{q}(a)=0$. If $a=\partial_{q+1}\left(a^{\prime}\right)$ for some $a^{\prime} \in C_{q+1}$ then $a$ is called a $q$-boundary. We denote the groups of $q$-cycles and $q$-boundaries by $Z_{q}$ and $B_{q}$ respectively, and define $Z_{0}=C_{0}$. Since $B_{q} \subseteq Z_{q}$, define the $q$ th homology group to be the quotient group $Z_{q} / B_{q}$, denoted by $H_{q}(\mathcal{C})$. Given $a \in Z_{q}$, the coset $a+B_{q}$ is the homology class in $H_{q}(\mathcal{C})$ determined by $a$. We denote this class by $[a]$.

Let $\mathcal{C}=\left\{C_{q}, \partial_{q}\right\}$ and $\mathcal{C}^{\prime}=\left\{C_{q}^{\prime}, \partial_{q}^{\prime}\right\}$ be two chain complexes. A chain map $f$ : $\mathcal{C} \rightarrow \mathcal{C}^{\prime}$ is a family of homomorphisms $\left\{f_{q}: C_{q} \rightarrow C_{q}^{\prime}\right\}$ such that $\partial_{q}^{\prime} f_{q}=f_{q-1} \partial_{q}$. A chain map $f: \mathcal{C} \rightarrow \mathcal{C}^{\prime}$ induces a homomorphism $f_{*}: H(\mathcal{C}) \rightarrow H\left(\mathcal{C}^{\prime}\right)$.

Let $K$ be a simplicial complex. A $q$-chain $a$ on $K$ is a formal sum of simplices of $K^{(q)}$. The $q$-chains form a group with respect to the component-wise addition mod 2; this group is the qth chain group of $K$, denoted by $C_{q}(K)$. The boundary of a $q$-simplex $\sigma=\left\langle v_{0}, \ldots, v_{q}\right\rangle$ is the formal sum: $\partial_{q}(\sigma)=\sum_{i=0}^{q}\left\langle v_{0}, \ldots, \hat{v}_{i}, \ldots, v_{q}\right\rangle$ where the hat means that $v_{i}$ is omitted. By linearity, the boundary operator $\partial_{q}$ can be extended to $q$-chains. The homology of $K$, denoted by $H(K)$, is defined by the homology of the chain complex $C(K)$.

Let $\mathcal{C}=\left\{C_{q}, \partial_{q}\right\}$ be a chain complex. Define the $q$-dimensional cochain group of $\mathcal{C}$ by the equation: $C^{q}(\mathcal{C})=\left\{c: C_{q} \rightarrow \mathbf{Z}_{2}\right.$ such that $c$ is a homomorphism $\}$. The boundary operator $\partial_{q+1}$ on $C_{q+1}$ induces the coboundary operator $\delta_{q}: C^{q} \rightarrow$ $C^{q+1}$ via $\delta_{q} c=c \partial_{q+1}$. It follows that $\delta_{q} \delta_{q-1}=0$. In the obvious way, there are also the dual notions of cocycles, coboundaries and cohomology of a cochain complex $C^{*}(\mathcal{C})$.

Given a simplicial complex $K, C^{q}(K)$ denote the $q$-cochain group $C^{q}(C(K))$. Observe that a $q$-cochain $c$ can be defined on the $q$-simplices of $K$ and it is naturally extended to $C_{q}(K)$. Define the cup product $\smile: C^{p}(K) \times C^{q}(K) \rightarrow$ $C^{p+q}(K)$ by the formula $\left(c \smile c^{\prime}\right)(\sigma)=c\left\langle v_{0}, \ldots, v_{p}\right\rangle \bullet c^{\prime}\left\langle v_{p}, \ldots, v_{p+q}\right\rangle$, where $\sigma=\left\langle v_{0}, \ldots, v_{p+q}\right\rangle \in K^{(p+q)}$. It induces an operation $\smile: H^{p}(K) \times H^{q}(K) \rightarrow$ $H^{p+q}(K)$ that is bilinear, associative, independent of the ordering of the vertices of $K$ and topologically invariant as follows: $[c] \smile\left[c^{\prime}\right]=\left[c \smile c^{\prime}\right]$. 